\title{
PEIXES DO CANAL DE SANTA CRUZ - PERNAMBUCO - BRASIL
}

\author{
AIDA M. ESKINAZI (*)
}

Laboratório de Ciências do Mar da Universidade Federal de Pernambuco

\section{SYNOPSIS}

O Canal de Santa Cruz está localizado no Estado de Pernambuco, a cerca de $50 \mathrm{Km}$ ao Norte do Recife. Foram encontradas 81 espécies de teleósteos, principalmente eurialinos. No presente artigo sobre a distribuição das espécies, é dada uma lista sistemática acompanhada do nome comum, das estações de coleta, da variação de salinidade, do habitat, etc. de cada espécie. As coletas foram realizadas em várias estações regulares e nos viveiros de peixe da área durante o período 1966-1969. Determinações de salinidade e de temperatura foram realizadas simultâneamente com as coletas. A salinidade na superfície e no fundo do Canal variou entre $17,00 \%$ oo e $34,00 \%$ oo e a temperatura entre $25^{\circ} \mathrm{C}$ e $31^{\circ} \mathrm{C}$.

\section{INTRODUÇÃO}

Em 1966 o Laboratório de Ciências do Mar da UFPe, iniciou uma série de estudos no Canal de Santa Cruz, motivado pela importância econômica que apresenta esta área. Sendo um local onde a pesca é realizada de maneira bastante intensa, houve o interesse de se fazer o estudo da ictiofauna presente, não só sob o ponto de vista puramente taxonômico, como também ecológico.

Existem poucas informações sobre a ecologia dos peixes estuarinos do Nordeste brasileiro. Eskinazi (1970), apresenta uma lista de espécies desta região, sem contudo entrar em muitos

(*) Bolsista do Conselho Nacional de Pesquisas.

Trab. Oceanogr. Univ. Fed. Pe., Recife, 13 283-302, 1972 
detalhes sobre a ecologia. Neste presente artigo procurou-se dar uma distribuição das espéceis encontradas, segundo os fatores ecológicos, principalmente a salinidade.

\section{MATERIAIS E MÉTODOS}

As coletas foram realizadas durante o período de 1966-1969, em estações espalhadas ao longo da parte sul (Fig. 1), nos viveiros de peixes em comunicação direta ou indiretamente com - Canal, nos pequenos cursos d'água que percorrem o manguesal e na porção marginal, em geral lamacenta, entre a PMM e BMM. Também foram feitas visitas aos entrepostos de pesca situados em Itapissuma.

Em todas as estações foram recolhidos, paralelamente a coleta de peixes, dados hidrológicos, principalmente salinidade e temperatura.

O material de pesca utilizado foi redes de arrasto de praia e tarrafas, além de coleta manual.

\section{BREVES CONSIDERAÇÕES SOBRE O BIÓTIPO}

O Canal de Santa Cruz é um braço de mar que contorna a. Ilha de Itamaracá, está localizado ao norte de Pernambuco, a $50 \mathrm{Km}$ da cidade do Recife. Sua extensão é de cerca de $22 \mathrm{Km}$, com larguras variáveis até $1 \mathrm{Km}$ e profundidade em tôrno de 4 a 5 metros durante a maré baixa. Entre os rios que nele desembocam temos como principais, na parte norte, os rios $\mathrm{Ca}$ tuama, Carrapicho e Botafogo, na parte sul, os rios Igarassu e Paripe. Suas margens lamacentas são ocupadas pelo manguesal típico de regiões tropicais.

De acordo com os dados hidrológicos obtidos, o regime de salinidade pode ser considerado marinho-poliano. A salinidade apresenta uma variação anual de $17,00-34,00 \%$, sendo que de um modo geral é mais elevada na estação seca que no inverno. Durante os meses que correspondem à época das chuvas, a salinidade é sempre inferior a $24,00 \% / 00$.

A mesma variação sazonal vista na salinidade, ocorre com a temperatura da água, entretanto esta é sempre elevada, como não poderia deixar de ser em se tratando de região de clima tropical. A variação encontrada foi de $25^{\circ} \mathrm{C}-31^{\circ} \mathrm{C}$.

O quadro abaixo dá uma idéia geral da variação da salinidade e temperautra na superfície do Canal: 


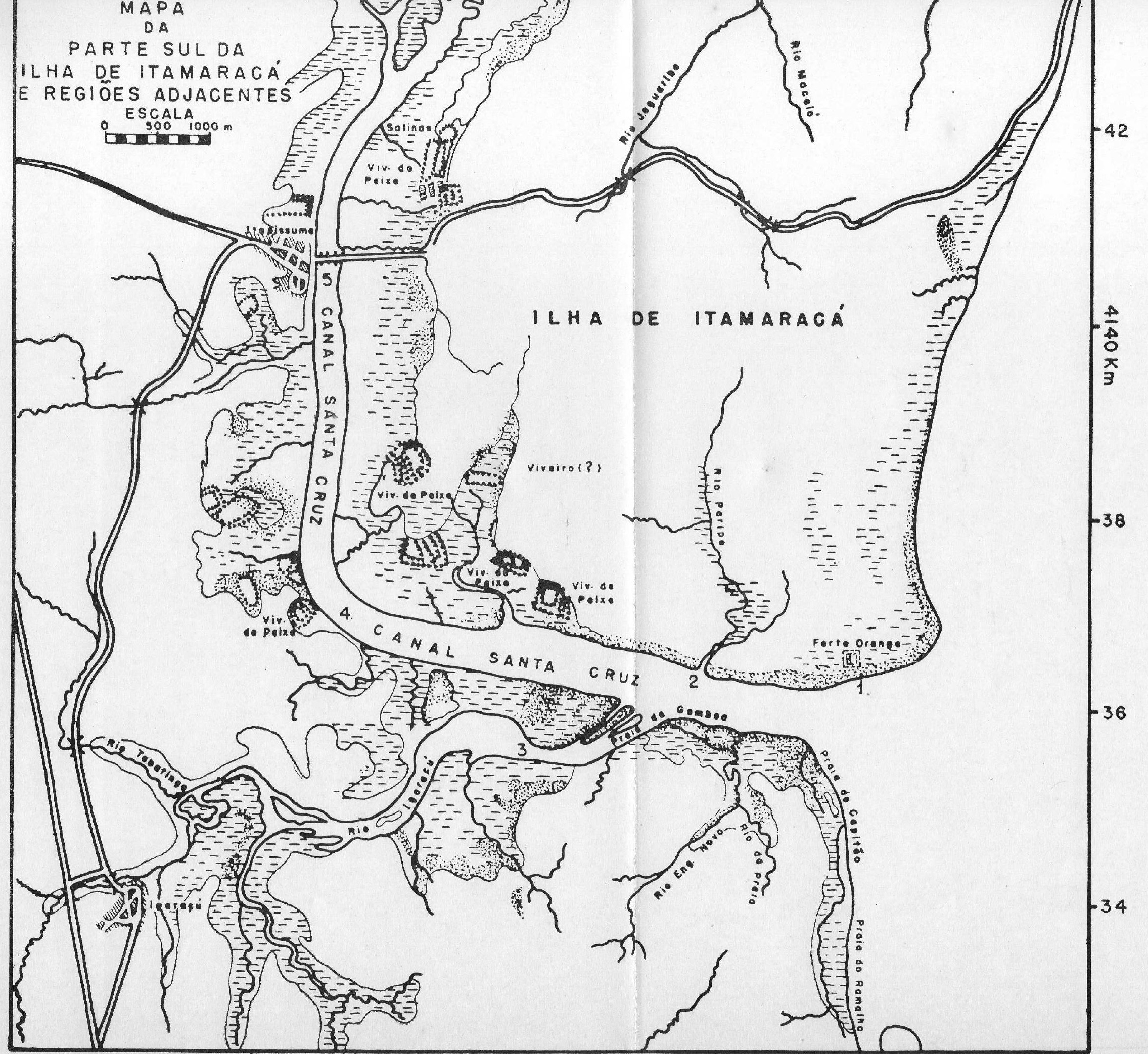


TEMPERATURA E SALINIDADE DA SUPERFICIE DAS AGUAS DO CANAL DE SANTA CRUZ (EST. 5) - JUNHO DE 1966 A SETEMBRO DE 1968, SEGUNDO COÊLHO, KOENING E RAMOS (NO PRELO).

Junho Set. Dez. Fev. Abril

TEMPERATURA $\left({ }^{\circ} \mathrm{C}\right)$

$\begin{array}{llllll}\text { MAXIMA } & 28,80 & 29,50 & 30,60 & 31,00 & 31,00 \\ \text { MINIMA } & 25,50 & 26,00 & 28,00 & 29,00 & 28,90\end{array}$

SALINIDADE $(\% / 00)$

\begin{tabular}{llllll} 
MAXIMA & 29,20 & 31,00 & 31,71 & 33,91 & 30,08 \\
MINIMA & 19,65 & 26,96 & 29,34 & 26,38 & 28,81 \\
\hline
\end{tabular}

Os viveiros de peixes apresentam acentuadas variações nos fatores hidrológicos, devido as condições precárias de funcionamento, acarretando assim uma considerável mortalidade nas espécies. A variação de salinidade foi de $0,23-54,88 \% / 00$, e da temperatura de $25^{\circ} \mathrm{C}-40^{\circ} \mathrm{C}$ (Silva 1970). Os indices mais elevados foram encontrados durante o período correspondente aos meses de verão.

\section{COMPONENTES E DISTRIBUIÇÃO DA ICTIOFAUNA}

A ictiofauna está representada por espécies tipicamente eurialinas, em sua grande maioria de origem marinha. A Tabela I apresenta uma lista das espécies distribuidas segundo a variação de salinidade encontrada. Para esta distribuição foram usados além dos dados registrados na região de estudo, aqueles encontrados em outros ambientes do Nordeste e os citados nas referências consultadas. A classificação dos regimes de salinidades usados na tabela seguiu a orientação dos trabalhos de Kiener (1965) e Coêlho (1967). Dentre os grupos encontrados, seis (6), o maior número de espécies pertence aquele marinho-polialino. Apenas duas (2) espécies são exclusivas de água salobra e doce, Dormitator maculatus e Colomesus psittacus. As espécies Oligoplites palometa e Eugerres brasilianus estão aqui colocadas como exclusivamente estuarinas apesar de pertencerem à família cuja maioria das espécies são de origem marinha; é que só foram utilizados para espécies os dados de salinidade registrados durante as coletas na área de estudo. Nesta Tabela I estão listadas apenas 76 espécies. 
TABELA I

\section{Espécies}

Megalops atlanticus

Elops saurus

Opisthonema oglinum

Harengula clupeoli

Lile piquitinga

Anchoo parvo

Poecilia vivipara

Sphyraena barracuda

Mugil curema

M. brasiliensis

M. trichodon

Centropomus undecimalis

C. parallelus

Oligoplites sourus sourus

Traquinotus falcatus

Eucinostomus gula

Eucinostomus argenteus

Gerres cinereus

Diapterus olisthosthomus

Gobionellus boleosoma

Citharichthys spilopierus Anchovia clupeoides

Tachysurus (selenaspis)herzbergi"

Xenomelaniris brasiliensis

Eucinosiomus pseudogula

Diapterus rhombeus

Bairdiella ronchus

Bathygobius soporator

Sphoeroides testudineus

Symphurus plagusia

Strongylura marina

Anchoo? hovelll
Distribuicão das especies segundo os Régimes de Solinidade

\begin{tabular}{|c|c|c|c|c|}
\hline & $\begin{array}{r}\text { bothat } \\
30_{0}\end{array}$ & $\begin{array}{l}18 \% \\
\text { MESOALINO }\end{array}$ & $\begin{array}{l}5 \% \\
\text { OLIGOALINC }\end{array}$ & \\
\hline
\end{tabular}




\begin{tabular}{|c|c|c|c|c|c|}
\hline \multirow{2}{*}{ Espécies } & \multicolumn{5}{|c|}{$\begin{array}{c}\text { Distribuicão das espécies segundo os Regimes } \\
\text { de Salinidade }\end{array}$} \\
\hline & \begin{tabular}{|r|}
$30 \%$ \\
MARINHO \\
\end{tabular} & $\begin{array}{c}30 \% \\
\text { POLIALINO } \\
\end{array}$ & \begin{tabular}{|l|}
$18 \%$ \\
MESOALINO
\end{tabular} & \begin{tabular}{|l|l|}
$5 \%$ \\
OLIGOALINOÁ
\end{tabular} & $\begin{array}{l}0,5 \% \\
\text { AGUA-DOOCE } \\
\text { AG }\end{array}$ \\
\hline Cetengraulis edentulus & & & & & \\
\hline Lycengraulis grossidens & & & & & \\
\hline Chloroscombrus chrysurus & & & & & \\
\hline Selene vomer & & & & & \\
\hline Eucinosiomus havana & & & & & \\
\hline Pomadasys corvinaeformI & & & & & \\
\hline Haemulon parral & & & & & \\
\hline Choefodipleurs faber & & & & & \\
\hline Gobionellus oceanicus & & & & & \\
\hline Anchoo tricolor & & & & & \\
\hline Gymnoforax nigromargina & & & & & \\
\hline Hyporhamphus unifasciatus & & & & & \\
\hline Hippocampus hudsonius & & & & & \\
\hline Fistularia tabacaria & & & & & \\
\hline Lutjonus apodus. & & & & & \\
\hline$L$ synagris & & & & & \\
\hline Lutjanus sp. & & & & & \\
\hline Ocyurus chrysurus & & & & & \\
\hline Anisotremus virginicus & & & . & & \\
\hline Chaetodon striatus & & & & & \\
\hline c. ocellatus & & & & & \\
\hline Abudefdut saxatills & & & & & \\
\hline Pomacentrus variabilis & & & & & \\
\hline Acanthurus bahianus & & & & & \\
\hline Erotelis smaragdus smaras & & & & & \\
\hline Gobionellus smaragdus & & & & & \\
\hline Prionotus alipionis & & & & & \\
\hline Dactylopterus volitans & & & & & \\
\hline Syacium micrurum & & & & & \\
\hline Bothus ocellatus & & & & & \\
\hline Chilomycterus & & & & & \\
\hline
\end{tabular}




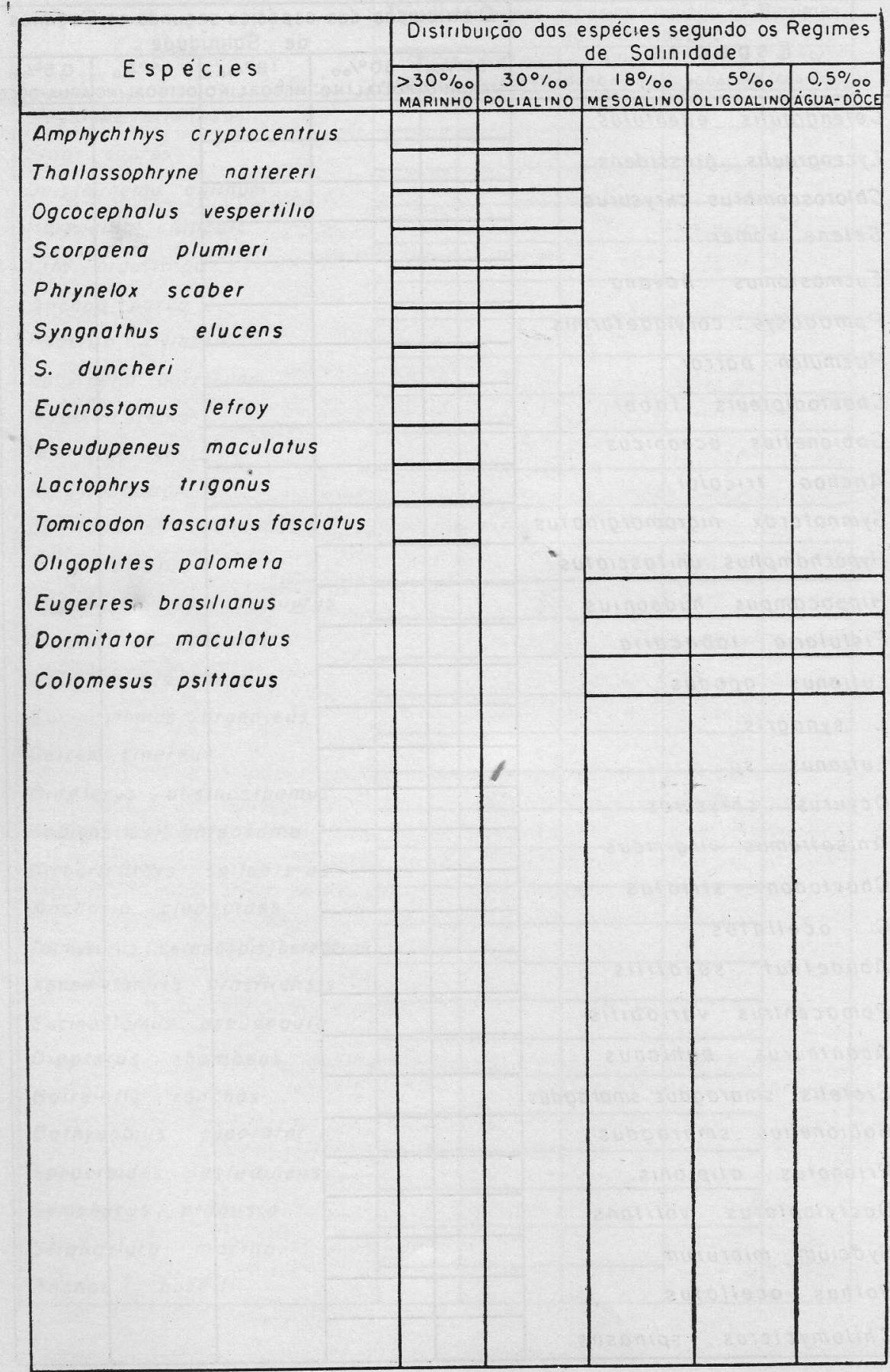




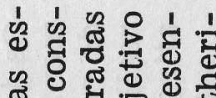

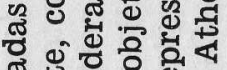

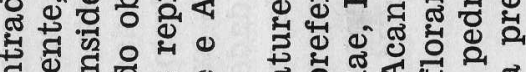

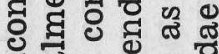
द्व

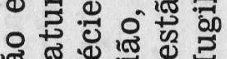

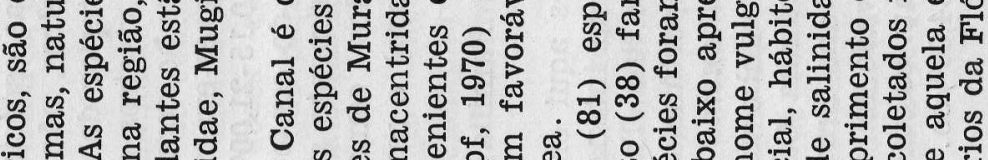

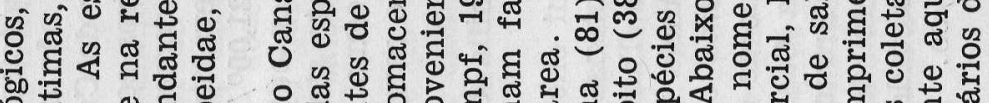

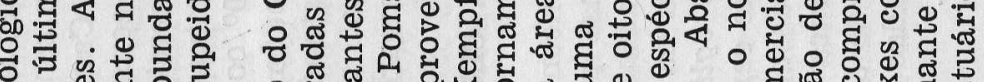

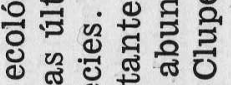
ब

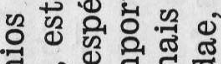
青 दี

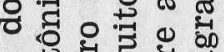

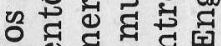
\&

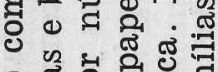
윤

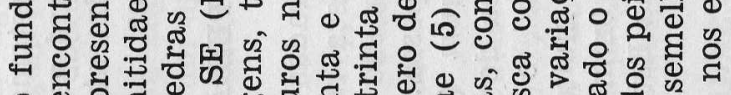

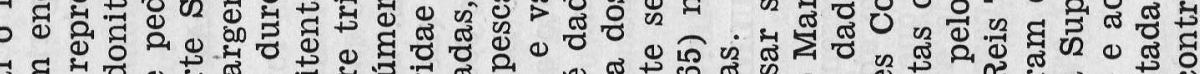

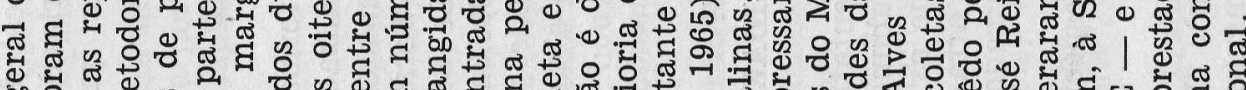

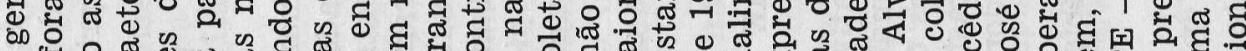
『 बै 車 ‡్丶 ๘

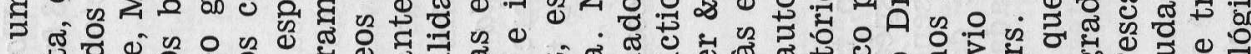
(c)

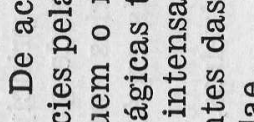

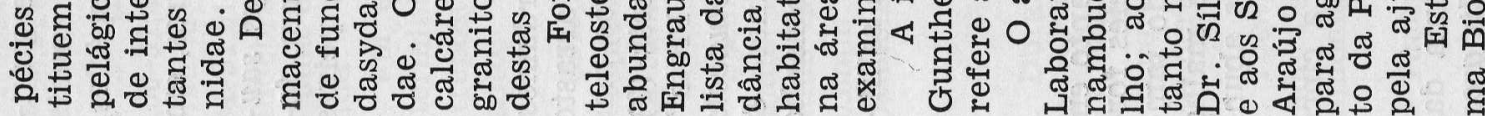


Os jovens destas espécies são bastantes abundantes, principalmente nos viveiros, não sendo observados exemplares de grande porte no interior do Canal. Entretanto nas pescarias anuais realizadas nos viveiros constituem uma das principais espécies, atingindo tamanho comercial.

Os exemplares examinados foram coletados em viveiros, em salinidade de $0,15-21,00 \%$ oo.

No exame do conteúdo estomacal de jovens de Elops saurus com comprimento standard variando de $137,0-173,0 \mathrm{~mm}$, foram encontrados como alimentos principais insetos, peixes (Poecilia vivipara) e camarões.

\section{Família CLUPEIDAE}

As espécies aqui encontradas formam pequenos cardumes, sendo objetos de intensa pesca realizada com rede de arrasto de malha muito fina. Também são encontradas nos viveiros, mas aí não têm impọrtância na pesca. Foram encontradas três (3) espécies: Opisthonema oglinum (Le Suer), "Sardinha Preta", atingindo o tamanho de cerca de $300,0 \mathrm{~mm}$; Harengula clupeola (Cuvier), "Sardinha Cascuda", e Lile piquitinga Schreiner \& Ribeiro, "Sardinha Branca", muito abundante, mas o tamanho máximo observado foi de $80,0 \mathrm{~mm}$.

Todas foram coletadas na Est. 5 e em viveiros, com salinidade de $0,15-34,00 \%$.

\section{Família ENGRAULIDAE}

Constitui uma das mais representativas da região, tanto em número de espécies (7), como em abundância. As espécies formam cardumes mais compactos que os Clupeideos, sendo também objetos de intensa pesca de arrasto com rede de malha fina. Segundo informações dos pescadores são pescadas abundantemente durante todo 0 ano.

Foram encontradas sete (7) espécies pertencentes a cinco (5) gêneros:

Anchovia clupeoides (Swainson), "Bôca Torta", é uma das espécies mais abundantes, e juntamente com Lycengraulis grossidens (Cuvier), atingem o maior tamanho entre os Engraulideos, atingindo o comprimento de $200,0 \mathrm{~mm}$. Coletado na Est. 5 e nos viveiros, em salinidade de $4,90-32,25^{\circ} / 00$.

Das três (3) espécies de Anchoa que frequentam a área, $A$. tricolor (Agassiz) "Tungão" e A. januaria Steindachner, "Rabo de Fogo", são as mais frequentes, enquanto que $A$. filifera (Fowler) Hildebrand parece pouco frequente, foram examinados 
apenas quatro exemplares encontrados no entreposto de pesca de Itapissuma e quatro (4) coletados em viveiros, salinidade de $7,94-8,21 \%$ oo.

A. tricolor foi coletado na Est. 5, salinidade observada de 29,00-31,31\%/00. Atinge cerca de $90,0 \mathrm{~mm}$ de comprimento. $A$. januaria, coletada na Est. 5 e viveiros, salinidade registrada 7,94-31,31\% $/ 00$. encontrada em águas doces da Venezuela (Hildebrand, 1963) e diz Cervigon (1966) que parece se tratar de uma espécie típica de águas doces ou salobras mais que marinha.

Foi encontrada apenas uma espécie de Anchoviella, não determinada, representada por um único exemplar recolhido no entreposto de pesca de Itapissuma, com $67,0 \mathrm{~mm}$ de comprimento.

Cetegraulis edentulus (Cuvier), é a única espécie do gênero representada para o Brasil. Coletada na Est. 5 e viveiros, com salinidade de $10,32-31,00 \%$ oo.

Lycengraulis grossidens (Cuvier), é um dos maiores Engraulideos da região, atingindo $200,0 \mathrm{~mm}$ de comprimento. No exame do conteudo estomacal de exemplares com 116,0 a $146,0 \mathrm{~mm}$ de comprimento, houve predominância de peixes, principalmente do gênero Eucinostomus, e camarões (Penaeus).

\section{Família BAGRIDAE}

Durante os arrastos feitos nas margens do Canal para coleta de material de estudo, não foram coletados espécimens desta família. As espécies aqui citadas foram encontradas no entreposto de pesca de Itapissuma, e segundo informações dos pescadores elas são mais freqüentes nas entradas do Canal.

Tachysurus (Selenaspis) herzbergii (Bloch), "Maruá"; segundo Cervigon (1966), esta espécie pode ser encontrada em salinidade de 1,021 até $35,00^{\circ} / 00$.

Tachysurus parkeri (Traill). "Bagre Amarelo".

Tachysurus proops (Valenciennes). "Bagre Branco" e "Ariassu".

\section{Família MURAENIDAE}

Examinada apenas uma espécie, Gymnotorax nigromarginatus (Girard), "Moréia"; muito comum nas margens lamacentas, 
em águas bastante rasas. Na Est. 4 quando a água está bem transparente, é vista facilmente em repouso sobre o fundo. $\mathrm{Pa}$ rece ser uma espécie típica de fundo mole, foi encontrada por Eskinazi \& Lima (1968) em fundo de areia e lama, em profundidade de 27,0 e $75,0 \mathrm{~m}$. Coletada na Est. 4, salinidade de 23,50 até $26,00^{\circ} / 00$.

Na Est. 5, na margem onde há vários blocos de pedras de granito, foi observada uma outra espécie de Muraenidae pescada com anzol. Ao exame superficial pareceu tratar-se de Gymnotorax funebris Ranzani, mas não foi possivel se conseguir exemplares para exame no Laboratório.

\section{Familia BELONIDAE}

Quando a maré começa a encher, pequenos cardumes de Belonidae são vistos na superfície das águas na parte mais profunda do Canal. Possivelmente trata-se de Strongylura marinc (Malbaum), "Agulhão", pois foi a unica espécie desta familia encontrada na área. Segundo Leccia (1965) é uma espécie que desce frequentemente aos rios.

Os exemplares examinados foram coletados em arrastos feitos na Est. 2.

\section{Família HAEMIRHAMPHIDAE}

Hyporhamphus unifasciatus (Ranzani), "Agulha Branca", os exemplares examinados foram recolhidos no entreposto de pesca de Itapissuma, mas não constituem objeto especial para a pesca.

\section{Família FISTULARIIDAE}

Fistularia tabacaria Linneaus, "Trombeta", é uma espécie pouco freqüente na região. Examinados dois exemplares coletados na Est. 4, salinidade observada de 27,92-31,44\% $/ 00$.

\section{Família SYNGNATHIDAE}

Foram encontradas três (3) espécies, todas coletadas em arrastos junto com muitas algas. O pouco material coletado não permitiu uma identificação muito segura das espécies.

Hippocampus? hudsonius punctulatus Guichenot, "Cavalo marinho", dois (2) exemplares coletados na Est. 2, salinidade registrada $32,00-35,00 \%$ oo. 
Syngnathus? elucens (Poey), um exemplar com $70,0 \mathrm{~mm}$ de comprimento standard, coletado na Est. 1.

Syngnathus duncheri Metzelaar, um exemplar com 37,0 mm de comprimento standard, coletado na Est. 1.

\section{Familia POECILIIDAE}

Poecilia vivipara Bloch \& Schneider, "Guaru", "Tin Tin", é uma espécie muito abundante, principalmente nos pequenos canais de maré que percorrem o manguesal e nos viveiros. É um tipo eurialino completo, podendo indiferentemente se reproduzir nos diversos meios que freqüentam (Kiener, 1965). Foi coletada nos viveiros em salinidade mínima de $0,15 \%$ e no Canal até 34,67\% \%o. Parece ocupar lugar de importância na alimentação de outros peixes, no exame do conteúdo estomacal de jovens de Centropomus e de Elops saurus, foi a espécie de peixe encontrada com maior frequiência. Atinge, na área o tamanho de $60,0 \mathrm{~mm}$.

\section{Família SPHYRAENIDAE}

Sphyraena barracuda (Walbaum), "Bicuda", bastante encontrada nos viveiros atingindo aí tamanho comercial. No interior do Canal só são encontrados indivíduos jovens. Não conseguem suportar à variação das condições ecológicas dos viveiros quando estes, em determinada época do ano, atingem as salinidades de $54,88^{\circ} / 00$ e a temperatura de $40^{\circ} \mathrm{C}$, é uma das primeiras espécies a morrer. Coletada na Est. 4 e viveiros, salinidade Observada $0,15-40,00 \%$ oo.

\section{Familia MUGILIDAE}

Constitui uma família típica para a região, está representada por três (3) espécies, Mugil curema Valenciennes, $M$. brasiliensis Agassiz e $M$. trichodon Poey. As espécies são multo abundantes e de grande importância econômica, constituindo as principais espécies de criação nos viveiros.

Mugil curema Valenciennes, "Tainha Parati", é a mais abundante das três espécies. Indivíduos muito pequenos, com cerca de 30,00 a $40,0 \mathrm{~mm}$, são também encontrados nos canais de maré que correm no manguesal.

As espécies "Tainha' 'e "Curimã" citadas por Schubart (1957), em seus estudos sobre viveiros do Recife-Pernambuco, possivelmente são $M$. curema e $M$. brasiliensis, respectivamente. Isto pelos caracteres por ele apresentados para separar as duas espécies, e por não ter a terceira espécie encontrada, $M$. trichodon, 
grande representação nos estuários e viveiros de Pernambuco. Segundo Schubart a "Tainha" cresce até $41,0 \mathrm{~cm}$ atingindo o peso de 710,0 gr., enquanto que a "Curimã" cresce mais e atinge $78,0 \mathrm{~cm}$ e $4.000 \mathrm{gr}$.

\section{Família ATHERINIDAE}

Quando a maré começa a encher, pequenos cardumes de Xenomelaniris brasiliensis (Quoy \& Gaimard) são vistos nas margens descendo o Canal e pequenos afluentes. Devido a sua abundância é alvo de uma pescaria bastante intensa juntamente com as espécies de Clupeidae e Engraulidae. Segundo Paiva de Caryalho (1954), desempenha papel importante na alimentação de outras espécies de maior porte. O tamanho máximo observado na região foi de $100,0 \mathrm{~mm}$. Coletada na Est. 2 e 5 nos viveiros, salinidade registrada de $5,00-34,00^{\circ} / 0$.

\section{- Família CENTRopomidaE}

As duas espécies encontradas, Centropomius undecimalis (Bloch) e C. parallelus Poey, estão incluídas entre as mais freqüentes e de importância econômica para a região. Encontram-se também bem adaptadas à vida nos viveiros.

Centropomus unäecimalis (Bloch), "Camurim Cabo de Machado", é a mais freqüente das duas espécies, atingindo o tamanho de $1,00 \mathrm{~m}$. É capaz de suportar grandes variações de salinidade (Gunther \& Hall, 1963). Foi encontrada em viveiro quando este atingiu a salinidade de $60,00^{\circ} / 00$, e também em salinidade de $0,15 \%$ oo. Fora das região de estudo, foi coletado um exemplar com cerca de $250,0 \mathrm{~mm}$, em água doce, no Rio Papocas no estado vizinho da Paraíba. Segundo ainda Gunther \& Hall é muito sensível à baixa de temperatura. Coletada nos Ests. 2,4 e 5 e viveiros.

Centropomus paralelus Poey, "Camurim Corcunda", é encontrado nas mesmas condições, sendo menos abundante.

\section{Familia CARANGIDAE}

Segundo Cervigon (1966), a maioria das espécie de Carangidae possuem duas fases bem definidas, uma juvenil claramente bentônica de fundo de areia e lama, e outra adulta pelágica. No Canal só são encontradas em estado jovem. Foram encontradas cinco (5) espécies. 
Traquinotus falcatus (Linnaeus), coletado em viveiros, salinidade registrada 0,15 a $10,59 \%$ oo.

Chloroscombrus chrysurus (Linnaeus), "Caracaxa", coletada na Est. 5 e viveiros, salinidade $7,94-30,00^{\circ} / 00$.

Selene vomer (Linnaeus), "Galo", Est. 4 e viveiros, salinidade $7,94-32,30^{\circ} / 00$.

Oligoplites saurus (Bloch \& Schneider), "Tibiro", Est. 4 e viveiros, salinidade $11,40-32,30^{\circ} / 00$.

Oligoplites palometa (Cuvier), "Tibiro", Est. 3a e viveiros, salinidade registrada 7,94-8,21\% \%o. Segundo Cervigon (1966), essta espécie é encontrada principalmente em água doce ou salobra.

\section{Família LUTJANIDAE}

Indivíduos jovens desta família são muito abundantes no Canal e são encontrados em grande quantidade durante as pescarias de arrastos feitas com redes de malha muito fina. Não são encontrados adultos, fazendo crer que as espécies encontradas são habitantes transitórios de águas salobras. Foram coletados quatro (4) espécies.

Lutjanus apodus (Walbaum), Est. 2, salinidade observada $23,00-32,00 \%$

L. synagris (Linnaeus), Est. 4 e 5 , salinidade registrada $17,00-21,00 \%$ oo.

Lutjanus sp., Est. 4, sem registro de salinidade.

Ocyurus crysurus (Bloch), Est. 5, sem registro de salinidade.

\section{Familia GERRIDAE}

E uma família típica da região, e a que se apresenta com o maior número de espécies (9). A maioria das espécies não atingem tamanho comercial, entretanto devido à sua abundância têm papel na pesca.

O gênero Eucinostomus está representado por cinco (5) espécies, e são conhecidas vulgarmente como "Carapicu". É muito difícil a separação das espécies de Eucinostomus principalmente entre $E$. gula (Cuvier) e $E$. argenteus Baird \& Girard. Segundo Gunther \& Hall (1963), E. gula é geralmente encontrada em sal1- 
nidades mais elevadas que $E$. argenteus. O registro de salinidade encontrada para estas duas espécies foi o seguinte: $E$. gula, coletados 40 exemplares nas Ests. 4, 5 e viveiros, com salinidade de 6,04 a $30,410 / 00 \mathrm{E}$. argenteus, vinte (20) indivíduos coletados apenas em viveiros, com salinidade de 6,04 a $11,18 \%$ oo. Leccia (1965) registra para a Venezuela, um exemplar de $E$. argenteus coletado em salinidade de $45,00^{\circ} / 00$, e Gunther \& Hall, 1965, dá para $E$. argenteus uma variação de salinidade de $0,2-32,2 \%$ oo.

As outras espécies de Eucinostomus encontradas foram:

E. pseudogula Poey, Est. 5 e viveiros, salinidade de ...... $2-, 58-20,43 \% / 00$.

E. Lefroy (Goode), Est. 1, salinidade $36,65 \%$ oo.

E. havana (Nichols), Est. 5, salinidade 17,29-32,55\% 00 .

Em dados estatísticos sobre a pesca, realizados no entreposto de pesca de Itapissuma, é comum encontrar a "Carapeba" entre as espécies comerciais. Foram encontradas quatro (4) espécies de Gerridae com este nome vulgar que são:

Gerres cinereus (Walbaum), coletada apenas em viveiros. Segundo Cervigón (1966) é encontrada desde a água doce ao mar aberto, atingindo tamanho comercial.

Diapterus olisthostomus (Good \& Bean) e D. rhombeus (Cuvier), coletados na Est. 5 e viveiros; salinidade registrada $5,17-20,61 \% / 00$. D. rhombeus atinge o tamanho de 40,0 cm.

Eugerres brasilianus (Cuvier), "Carapeba de Lista", coletada em viveiros, salinidade observada $0,21-8,01 \% / 00$.

\section{Família POMADASYIDAE}

Com três (3) espécies encontradas, pouco comuns e sem grande representação para a área.

Pomadasys corvinaeformis (Steindachner), Est. 3a, salinidade registrada $10,52-22,88 \%$.

Anisotremus virginicus (Linnaeus), Est. 5, salinidade ... $30,00-33,00 \%$ oo.

Haemulon parrai (Desmarest), Est. 4 e5, salinidade .... $17,29-32,30 \%$ oo.

\section{Família SCIAENIDAE}

Representada por uma única espécies Bairdiella ronchus (Cuvier), pouco freqüente e sem importância comercial. Foi coletada na Est. 5 e na entrada de um dos viveiros. Salinidade registrada, $2,00-34,00^{\circ} / 00$. 
Pseudupeneus maculatus (Bloch), muito rara. uma espécie de preferência de fundos rochosos. Foram examinados apenas dois (2) exemplares com $70,0 \mathrm{~mm}$ de comprimento standard, coletados na Est. 5.

\section{Família EPHIPPIDAE}

Chaetcipterus faber (Broussonet), espécie habitual nos arrastos feitos nas margens, mas geralmente é encontrado em estado juvenil, não excedendo a $80,0 \mathrm{~mm}$. Coletado na Est. 5 .

\section{Família CHAETODONTIDAE}

As duas espécies encontradas são muito raras na região, são de preferência de fundos rochosos e coralinos.

Chaetodon striatus Linnaeus e C. ocellatus Bloch, Est. 5, salinidade registrada, $27,92-32,00^{\circ} / 00$.

\section{Família POMACENTRIDAE}

Encontradas duas espécies, características de fundos rochosos e coralinos.

Abudefduf saxatilis (Linnaeus), examinado apenas um indlvíduo com 48,0mm de comprimento standard, coletado na Est. 4.

Pomacentrus variabilis Castelnau, apenas um exemplar coletado na Est. 3a.

\section{Família ACANTHURIDAE}

Uma espécie, Acanthurus bahianus Castelnau, característica de fundos rochosos, pouco encontrada na área. Examinado um exemplar com $45,0 \mathrm{~mm}$ coletado na Est. 4. Salinidade registrada $28,00-32,00 \% / 00$.

\section{Família ELEOTRIDAE}

Encontra-se muito bem representada na região, sendo as espécies muito abundantes. Duas espécies foram encontradas:

Dormitator maculatus (Bloch), "Cundunde", bastante abundante nos viveiros, salinidade registrada, 2,00-11,00\% $/ 00$. E uma espécie típica de águas doces e salobras.

Erotelis smaragdus smaragdus (Valenciennes) é muito comum em tocas feitas na lama, que ficam a descoberto durante a baixa mar. E encontrada neste ambiente junto com os peixes Gobionellus smaragdus (Valenciennes) e G. boleosoma (Jordan 
\& Gilbert), além de um camarão do gênero Alpheus e de outros crustáceos. Jordan \& Evermann (1898) referem-se a Erotelis smaragdus (Valenciennes) como estritamente marinha, encontrada em costas coralinas junto com algas verdes. Entretanto a outra subespécie $E$. smargdus civitatum (Ginsburg), é dada como coletada em lama mole profunda (Ginsburg, 1953), em habitat semelhante ao encontrado na nossa região.

Os exemplares foram coletados manualmente durante a maré baixa, nas Ests. 2 e 4 .

\section{Familia GOBIIDAE}

As espécies são muito abundantes nas margens lamacentas do Canal e afluentes. Foram encontradas quatro (4) espécies, Bathygobius soporator (Valenciennes), Gobionellus smaragdus (Valenciennes), G. boleosoma (Jordan \& Gilbert) e G. oceanicus (Pallas).

Indivíduos jovens de $B$. soporator são encontrados nas pequenas poças d'águas formadas nas margens durante a maré baixa. A espécie foi coletada na Est. 2,4 e 5 e viveiros, salinidade registrada de $6,00-32,00 \%$ oo.

Gerlach (1958) refere-se a Gobionellus smaragdus (Valenciennes), como encontrada cavando buracos na lama das margens do manguesal desprovidas de vegetação, vivendo aí junto com um camarão do gênero Alpheus. Aqui encontramos esta espécie, e mais Gobionellus boleosoma (Jordan \& Gilbert), em ambiente semelhante, em tocas na lama, acompanhado por um camarão Alpheus, além do peixe Erotelis smaragdus e outros crustáceos, formando uma associação bastante característica.

A outra espécie, Gobionellus oceanicus (Pallas), "Mororo", é muito comum nos arrastos realizados nas margens lamacentas. Coletada na Est. 5 e viveiros, salinidade de 7,00-31,00\%/00.

\section{Família SCORPAENIDAE}

Com uma só espécie, Scorpaena plumieri Bloch, muito rara. Coletados apenas dois (2) indivíduos na Est. 1 e 4.

\section{Família TRIGLIDAE}

Prionotus alipionis Teague \& Myers, coletado apenas um exemplar na Est. 1, com $88,0 \mathrm{~mm}$ de comprimento standard.

\section{Família DACTYLOPTERIDAE}

Dactylopterus volitans (Linnaeus), muito freqüente nas margens lamacentas. Coletada na Est. 4, sem registro de salinidade. 
As espécies são muito comuns nas margens, e são conhecidas vulgarmente por "Solhas" Foram encontradas três 3 espécies.

Citharichthys spilopterus Gunther, a mais freqüente, coletada nas Ests. 1, 4, 5 e viveiros, salinidade registrada ...... $11,00-33,00 \%$ oo.

Syacium micrurum Ranzani, Est. 4, salinidade de ..... $22,00-26,00 \%$ oo.

Bothus ocellatus (Agassiz), Est. 2, salinidade de ....... $27,00-34,00 \%$ oo.

\section{Família CYNOGLOSSIDAE}

Symphurus plagusia (Bloch \& Schneider), encontrada nas margens lamacentas. Foi registrada por Eskinazi \& Lima (1968) em fundo de lama e profundidade de $21,0-75,0 \mathrm{~m}$. Coletada na Est. 4, sem registro de salinidade.

\section{Família TETRAODONTIDAE}

Com duas espécies:

Colomesus psittacus (Bloch \& Schneider), coletado apenas um (1) indivíduo com $45,0 \mathrm{~mm}$ de comprimento standard, no viveiro, em salinidade $11,40^{\circ} / 00$.

Sphoeroides testudineus (Linnaeus), "Baiacu", é uma das espécies de maior freqüência nos arrastos realizados nas margens. Coletada nas Ests. 1, 2, 3, 4 e 5. Salinidade registrada, $20,00-34,00 \%$ oo.

\section{Família DIODONTIDAE}

Chilomycterus spinosus (Linnaeus), examinado apenas um exemplar com $82,0 \mathrm{~mm}$ de comprimento standard, encontrado no entreposto de pesca de Itapissuma.

\section{Família OSTRACIIDAE}

Lactophrys trigonus (Linnaeus), um exemplar com $66,0 \mathrm{~mm}$ de comprimento standard coletado na Est. 2, salinidade de .... $32,00-34,00 \% / 00$.

\section{Família GOBIESOCIDAE}

A espécie encontrada, Tomicodon fasciatus fasciatus (Peters) possui um habitat bastante característico. Um exemplar 
com $21,0 \mathrm{~mm}$ de comprimento standard foi encontrado dentro do tubo de um Teredo, na raiz de Rizophora a margem do mangue na Est. 1. E um outro indivíduo com o mesmo comprimento, em fendas nas pedras que ficam a descoberto durante a baixa mar na Est. 2.

\section{Família BATRACHOIDIDAE}

As duas espécies encontradas não são muito freqüentes na região.

Amphichtys cryptocentrus (Valenciennes), encontrada na parte mais profunda do Canal, cerca de $6,0 \mathrm{~m}$ de profundidade. Coletada na Est. 5, com anzol.

Thalassophryne nattereri Steindachner, ao contrário da espécie precedente, foi encontrada nas margens lamacentas das Ests. 2 e 5.

\section{Família OGCOCEPHALIDAE}

Ogcocephalus vespertitio (Linnaeus), "Peixe Morcêgo", coletado na Est. 4, salinidade 27,92-30,35\% 00 .

\section{Família ANTENARIIDAE}

Phrynelox scaber (Cuvier), pouco encontrada, 4 exemplares na Ests. 4 e 5.

\section{SUMMARY}

The Canal of Santa Cruz is located in the State of Pernambuco, about $50 \mathrm{~km} \mathrm{~N}$ of Recife. Eighty one (81) species of teleosteos fishes were found. The ichthyofauna is composed of typically euryhaline species. In this paper a study on the distribution of the species, a systematic list with the common name, stations of collect, range of salinity, habitat, etc., are given. The collections were made at various regular stations distributed on the Canal and in the fish ponds in the area, during the period 1966-1969. Measurements of salinity and temperature were also made in the stations. The range of superficie and botton salinities found in the Canal is between $17,000 / 00-34,00 \%$ oo and the temperature varied from $25,00-31,00^{\circ} \mathrm{C}$.

\section{REFERENCIAS}

CARVALHO, J. de P. Nótulas ictiológicas, III. Sobre a ocorrência de Xenomelaniris brasiliensis (Quoy \& Gaimard) no litoral do Estado de S. Paulo (Brasil) (Pisces Mugiloide-Atherinidae). Bol. Inst. Oceanogr. S. Paulo, 5 (1-2): 241--260, 1954. 
CERVIGON, F. M. Los peces marinos de Venezuela. I. Caracas, Fundacion La Salle de Ciências Naturales, 1966, 438 p.

. Ecologia Marina. Cap. 10. Los peces. Caracas, Fundacion La Salle de Ciências Naturales: 308-335, 1967.

COELHO, P.A. Os crustáceos decápodos de alguns manguezais pernambucanos. Trab. Inst. Oceanogr. Univ. Fed. Pernambuco, 7/8: 71-90, 1967.

COELHO, P. A., KOENING, M. L. \& RAMOS, M. de A. A macrofauna bêntica dos estuários de Pernambuco e da Paraíba. Anais IV Congresso Latinoamericano Zool., Caracas, 1968 (no prelo).

ESKINAZI, A. M. \& LIMA, H. H. Peixes marinhos do Norte e Nordeste do Brasil, coletados pelo Akaroa, Canopus e N. Oc. Almirante Saldanha. Arq. Est. Biol. Mar. Univ. Fed. Ceará, Fortaleza, 8 (2): 163-172, 1968.

ESKINAZI, A. M. Lista preliminar dos peixes estuarinos de Pernambuco e Estados vizinhos (Brasil). Trab. Oceanogr. Univ. Fed. Pernambuco 9/11: 265-274, 1970.

GERLACH, S. A. Mangroveregion tropischer Kusten als Lebensraum. Zeitsch Morph. Okol. Tiere, 46: 636-730, 1958.

GINSBURG, I. Ten new american Gobioid Fishes in the United States National Museum, including additions to a revision of Gobionellus. Journal of the Washington Acad. Sci., 43 (1): 18-26, 1953.

GUNTHER, G. \& HALL, G. E. Biological investigation of St. Lucie Estuary (Florida) in connection with Lake Okeechobee discharges through the St. Lucie Canal. Gulf Coast Res. Lab., 1 (5): 189-307, 1963.

A biological investigation of the Caloosahatches Estuary of Florida. Gulf Coast Res. Lab., 2(1): 1-71, 1965.

HILDEBRAND, S. F. Family Engraulidae. In: Yale University Sears Foundation for Marine Research, memoir Fishes of the Western North Atlantic 1. New Haven, 1968.

JORDAN, D. S. \& EVERMANN, B. W. The Fishes of North and Middle America: a descriptive catalogue of the species of fishlike vertebrates found in the waters of North America, North of the Isthmus of Panamá. Bull. U. S. Nat. Mus., Washington, 47, Pt. III: 2183-3134, 1898.

KEMPF, M. Nota preliminar sobre os fundos costeiros da região de Itamaracá (Norte do Estado de Pernambuco, Brasil). Trab. Oceanogr. Univ. Fed. Pernambuco, 9/11: 95-110, 1970.

KIENER, A. Contribution à l'étude écologique et biologique des eaux sumatres malgaces. Les poissons euryalins et leur rôle dans le developpment des pêches. Vie et Millieu, XVI (2-C): 103-1149. 1965.

Trab. Oceanogr. Univ. Fed. Pe., Recife, 13 283-302, 1972 
LECCIA, F. M. Contribution a la sistematica y ecologia de los peces de la Laguna de Unare, Venezuela. Bul. Mar. Sci., 15(2): 274330,1965 .

SCHUBART, O. Investigações sobre os viveiros do Recife. In Problemas da pesca no Nordeste e em Pernambuco. Série Política Eco-

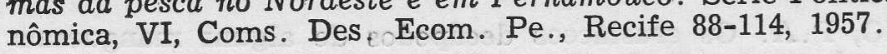

SILVA, J.E. Nota prévia sobre viveiros de peixes situados em Itamaracá-Pernambuco (Brasil). Trab. Oceanogr. Univ. Fed. Pernambuco, 9/11: 317-324; 1970. 